\title{
Canonical partition function for the hydrogen atom in curved space
}

\author{
S.M. Blinder \\ Department of Chemistry, University of Michigan, Ann Arbor, MI 48109-1055, USA
}

Received 27 June 1995

\begin{abstract}
The electronic partition function for the hydrogen atom was recently derived by integration over the Coulomb propagator. A much simpler derivation is given here, based on Schrödinger's exact solution for a hydrogenic atom in a Riemannian space of positive curvature. The energy spectrum is entirely discrete, including states which correspond to the ionized atom. The curvature in Riemannian space is shown to be equivalent to a finite volume in Euclidean space.
\end{abstract}

In a recent publication we evaluated the electronic partition function for the hydrogen atom by integration over the Coulomb propagator [1]. This is a conceptually straightforward way of avoiding the apparent divergence of the partition function which a naive sum over states might imply. In this paper, we present a much simpler derivation of essentially the same result.

Schrödinger in 1940 obtained an exact solution of the wave equation for a hydrogen atom in a Riemannian space of constant positive Gaussian curvature [2]. We must emphasize from the outset that the actual curvature of space-time in our corner of the Universe is far too feeble to have any perceptible effects on atomic structure. We will show that Riemannian curvature can be regarded as a metaphor for representing a finite volume in 3-dimensional Euclidean space.

For a hydrogenlike atom in a space of positive curvature $1 / R$, Schrödinger showed that the energy eigenvalues are given by a remarkably simple formula:

$$
E_{n}=-\frac{Z^{2}}{2 n^{2}}+\frac{\left(n^{2}-1\right)}{2 R^{2}}, \quad n=1,2,3 \cdots
$$

with the familiar Coulomb degeneracies

$$
g_{n}=n^{2} .
$$

We are using atomic units $\hbar=m=|e|=1$ and assuming infinite nuclear mass. Distances are accordingly expressed in bohrs $\left(a_{0}=\hbar^{2} / m e^{2}\right)$ and energies in hartrees $\left(E_{\mathrm{H}}=e^{2} / a_{0}\right)$. We also define the dimensionless temperature parameter 


$$
\beta \equiv E_{\mathrm{H}} / k T=315774 / T
$$

with $T$ in kelvins. The two terms of eq. (1) have the forms, respectively, of a Coulombic energy plus a free-particle energy. It would be difficult to imagine a formula with any simpler structure.

The hydrogen atom in positively-curved space has a purely discrete energy spectrum, in contrast to Euclidean hydrogen, which also possesses a continuum. The ground state energy is $E_{1}=-Z^{2} / 2$, same as for the Euclidean 1s state. For the following value of the principal quantum number:

$$
n_{0} \approx \sqrt{Z R},
$$

the energy is approximately equal to zero. We will find $n_{0} \sim 10^{5}$. This can be considered the dividing line between negative-energy bound and positive-energy ionized or "free" hydrogenic states. Note that, in contrast to the Euclidean case, the positive-energy states have a discrete, albeit extremely dense, eigenvalue spectrum.

We can now write down the electronic partition function, based on $E_{1}$ as the energy origin:

$$
\begin{aligned}
q_{\mathrm{elec}} & =\sum_{n=1}^{\infty} n^{2} e^{-\beta\left(E_{n}-E_{1}\right)} \\
& =1+e^{-\beta Z^{2} / 2} \sum_{n=2}^{\infty} n^{2} e^{\beta Z^{2} / 2 n^{2}} e^{-\beta\left(n^{2}-1\right) / 2 R^{2}} .
\end{aligned}
$$

Setting $Z=0$ reduces eq. (5) to a free-electron partition function:

$$
q_{0}=\sum_{n=1}^{\infty} n^{2} e^{-\beta\left(n^{2}-1\right) / 2 R^{2}} \approx \int_{0}^{\infty} n^{2} e^{-\beta n^{2} / 2 R^{2}} d n=\frac{\sqrt{\pi} R^{3}}{2 \beta^{3 / 2}} .
$$

Identifying this with the free-particle partition function

$$
q_{0}=(2 \pi m k T)^{3 / 2} V / h^{3}=(2 \pi \beta)^{-3 / 2} V,
$$

we can associate the volume $V$ in Euclidean space with the radius of curvature $R$ in Riemannian space as follows:

$$
V=2 \pi^{2} R^{3} .
$$

(The same formula also relates the hypersurface area of a 4-dimensional hypersphere to the radius $R$ ). The above correspondence leads us to interpret the curvature as a metaphor for a finite volume in Euclidean space, whereby

$$
R=\left(V / 2 \pi^{2}\right)^{1 / 3} .
$$

Laboratory-size volumes correspond to radii $R$ of the order of $10^{10}$ bohrs.

We express the hydrogenic partition function in the form

$$
q_{\text {elec }}=q_{\text {bound }}+q_{\text {free }} e^{-\beta Z^{2} / 2} \text {. }
$$




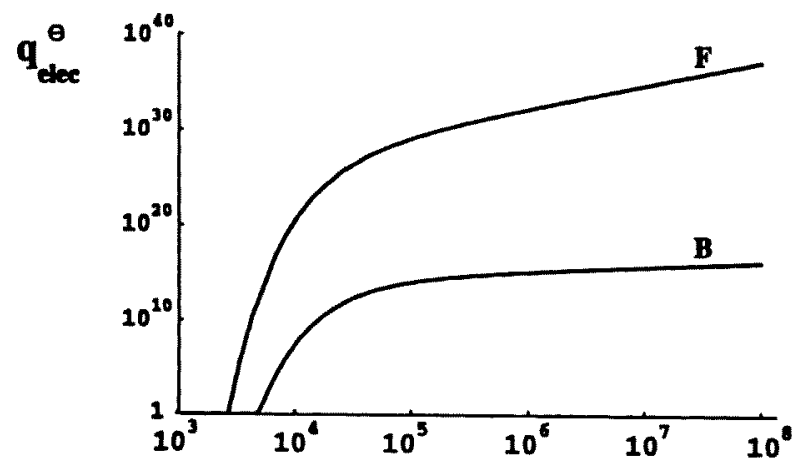

$\mathbf{T}(\mathbf{K})$

Fig. 1. Bound (B) and free (F) contributions to the standard-state hydrogen atom partition function.

Thus defined, the bound part is based on the ground state as energy origin, $E_{1} \equiv 0$, while the free part uses $E\left(n_{0}\right) \equiv 0$.

We evaluate the indefinite integral

$$
\begin{aligned}
f(x) \equiv & \int x^{2} \exp \left[\frac{\beta Z^{2}}{2 x^{2}}-\frac{\beta x^{2}}{2 R^{2}}\right] d x=-\frac{R^{2} x}{\beta} \exp \left[\frac{\beta Z^{2}}{2 x^{2}}-\frac{\beta x^{2}}{2 R^{2}}\right] \\
& +\left\{\frac{\sqrt{\pi}}{2 \sqrt{2} \beta^{3 / 2}}\left(R^{3}-i \beta Z R^{2}\right) e^{i \beta Z / R} \operatorname{erf}\left[\sqrt{\frac{\beta}{2}}\left(\frac{x}{R}+i \frac{Z}{x}\right)\right]+\text { C.C. }\right\} .
\end{aligned}
$$

For some special values of $x$ :

$$
\begin{aligned}
& f(\infty)=\frac{\sqrt{\pi} R^{3}}{2 \beta^{3 / 2}}=(2 \pi \beta)^{-3 / 2} V=q_{0}, \\
& f\left(n_{0}\right)=f(\sqrt{Z R}) \approx \frac{1}{3}(Z R)^{3 / 2} .
\end{aligned}
$$

We note in addition that $f\left(n_{0}\right) \gg f\left(n^{*}\right)$ for $n_{0} \gg n^{*} \gg 1$.

By approximating summations over slowly-varying terms by integrals we obtain

$$
\begin{aligned}
q_{\text {bound }} & \approx 1+e^{-\beta Z^{2} / 2}\left[\sum_{n=2}^{n^{*}} n^{2} e^{\beta Z^{2} / 2 n^{2}} e^{\beta n^{2} / 2 R^{2}}+f\left(n_{0}\right)-f\left(n^{*}\right)\right] \\
& \approx 1+\frac{1}{3}(Z R)^{3 / 2} e^{-\beta Z^{2} / 2}
\end{aligned}
$$

since the parts involving $n^{*}$ are negligible compared to either 1 or $f\left(n_{0}\right)$ at all temperatures. Also

$$
q_{\text {free }} \approx f(\infty)-f\left(n_{0}\right) \approx q_{0}
$$

since $f\left(n_{0}\right)$ is negligible compared to $q_{0}$. 
The two contributions to the electronic partition function for hydrogen are plotted in fig. 1. These are referred to the thermodynamic standard state, with volume given by

$$
V^{\theta}=N_{\mathrm{A}} k T / p^{\theta}=5.5375 \times 10^{26} T \mathrm{bohr}^{3}
$$

with $p^{\theta}=1$ bar. The curves labelled $\mathrm{B}$ and $\mathrm{F}$ represent, respectively, $q_{\mathrm{bound}}^{\theta}$ and $q_{\mathrm{free}}^{\theta} e^{-\beta / 2}$. Visually, these curves are indistinguishable from their analogs obtained via the Coulomb propagator in ref. [1].

\section{References}

[1] S.M. Blinder, J. Math. Phys. 36 (1995) 1208. This paper contains references to earlier work on the hydrogen partition function.

[2] E. Schrödinger, Proc. Irish Acad. A46 (1940) 9;

see also: N. Bessis and G. Bessis, J. Phys. A. Math. Gen. 12 (1979) 1991. 